\title{
Power means with integer values
}

\author{
Ralph Høibakk and Dag Lukkassen
}

\begin{abstract}
Ralph Høibakk obtained his Master degree in physics from the Norwegian Institute of Technology in 1962. He is now a part-time professor at Narvik University College within Enterprise Development.

Dag Lukkassen obtained his Dr. Scient. degree in mathematics from Troms $\varnothing$ University in 1996. He is a full professor of mathematics at Narvik University College since 2000. His main field of interest is the theory of partial differential equations and their applications.
\end{abstract}

Throughout this paper $a$ and $b$ will denote positive real numbers. The $k$-th power mean $P_{k}=P_{k}(a, b)$ (with equal weights) of $a$ and $b$ is defined by

$$
P_{k}= \begin{cases}\left(\frac{a^{k}+b^{k}}{2}\right)^{1 / k} & \text { if } k \neq 0, \\ \sqrt{a b} & \text { if } k=0 .\end{cases}
$$

The most well-known power means are the arithmetic, the geometric and the harmonic mean given by the formulae

$$
A=\frac{a+b}{2}, \quad G=\sqrt{a b}, \quad H=\frac{2 a b}{a+b},
$$

respectively. For more detailed information on power means, see e.g. the books [3] and [7].

Das $k$-te Potenzmittel $P_{k}(a, b)$ zweier positiver reeller Zahlen $a, b$ ist für $k \neq 0$ durch $\left(\left(a^{k}+b^{k}\right) / 2\right)^{1 / k}$ bzw. im Fall $k=0$ durch $\sqrt{a b}$ gegeben. In der vorliegenden Arbeit gehen die beiden Autoren der Frage nach, für welche Paare $(a, b)$ natürlicher Zahlen das Mittel $P_{k}(a, b)$ ebenfalls ganzzahlig ist. Unter Verwendung eines Ergebnisses von H. Darmon und L. Merel aus dem Jahr 1997, das seinerseits auf dem Beweis der Fermat-Vermutung durch A. Wiles beruht, beweisen sie, dass für $|k|>2$ keine solchen Zahlenpaare $(a, b)$ existieren können. In den verbleibenden Fällen $k=0, \pm 1, \pm 2$ geben die Autoren eine vollständige Klassifikation der möglichen Zahlenpaare $(a, b)$ mit der gewünschten Eigenschaft. 
In this paper we consider the problem of determining integers $a$ and $b$ such that the corresponding power mean $P_{k}$ becomes integer valued. For integer values of $k$ satisfying $|k| \geq 3$ the answer follows easily by the following variant of Fermat's Last Theorem. We recall that a solution $(x, y, z)$, where $\operatorname{gcd}(x, y, z)=1$, is called trivial if $x y z=0$ or \pm 1 , and is called non-trivial otherwise.

Theorem 1. The equation $x^{n}+y^{n}=2 z^{n}$ has no non-trivial solution for integer values of $n \geq 3$.

This result was conjectured and even proved for $2<n<31$ by Denés [6] in 1952 . Motivated by Wiles' famous breakthrough in the early 90's, which led to the proof of Fermat's Last Theorem, Ribet [10] proved the theorem when $n$ is divisible by a prime which is congruent to $1 \bmod 4$. By retracing the steps in Ribet's argument, Darmon and Merel [4] were able to give the final proof of the theorem in 1997.

Thanks to this theorem we can easily verify the following result:

Corollary 2. If $a \neq b$ are integers and $k$ is an integer satisfying $|k| \geq 3$, then $P_{k}$ is not an integer.

Proof. The case $k \geq 3$ follows directly from Theorem 1 by putting $n=k, x=a, y=b$ and $z=P_{k}$. For $k \leq-3$ we put $n=-k$. Hence, by rearranging, we obtain that

$$
a^{n}+b^{n}=2\left(\frac{a b}{P_{k}}\right)^{n} .
$$

Therefore, if $P_{k}$ is an integer, so is $a b / P_{k}$. But this contradicts Theorem 1 for $x=a$, $y=b$ and $z=a b / P_{k}$. Hence, the proof is complete.

It is certainly easy to find all integers making the arithmetic mean or the geometric mean integer valued. Concerning the harmonic mean we are going to prove the following result:

Theorem 3. The integers $a$ and $b$ making the harmonic mean integer valued are precisely those of the form

$$
a=t p(p+q), \quad b=t q(p+q)
$$

(in this case $H=2 t p q$ ) and the form

$$
a=t(2 p+1)(p+q+1), \quad b=t(2 q+1)(p+q+1)
$$

(in this case $H=t(2 p+1)(2 q+1)$ ), where $p, q$ and $t$ are positive integers.

Proof. The fact that $H$ is an integer when $a$ and $b$ are of the forms (2) and (3) is seen directly by inspection. It remains to prove that $H$ is an integer only if $a$ and $b$ are of these forms. Assume that $H$ is an integer and let $r$ and $s$ be the integers given by

$$
r=2 a-H, \quad s=2 b-H .
$$

From the identity $H=2 a b /(a+b)$ we obtain the simple relation

$$
H^{2}=r s \text {. }
$$


Next we put $r=w r_{1}, s=w s_{1}$, where $w=\operatorname{gcd}(r, s)\left(\operatorname{such}\right.$ that $\left.\operatorname{gcd}\left(r_{1}, s_{1}\right)=1\right)$. By (5) we see that $r_{1} s_{1}$ is a perfect square, and since $r_{1}$ and $s_{1}$ do not have any prime factors in common, $r_{1}$ and $s_{1}$ have to be perfect squares, i.e. of the forms $r_{1}=k^{2}, s_{1}=l^{2}$. Thus, by (4) and (5) we find that

$$
2 a=k w(k+l), \quad 2 b=l w(k+l) .
$$

Investigating the only two possible combinations

1. $(k, l)=(2 p+1,2 q+1)$ and $w=t$,

2. $(k, l)=(p, q)$ and $w=2 t$,

where $p, q$ and $t$ are integers, we arrive at the two possible representations (2) and (3). This completes the proof.

Remark 4. Let $t=1$ and put $p=1$ and $p=0$ in (2) and (3), respectively. Then the above result shows that

$$
H= \begin{cases}2 q & \text { if } a=1+q, b=q(1+q), \\ 2 q+1 & \text { if } a=1+q, b=(2 q+1)(1+q) .\end{cases}
$$

This formula shows that $H$ takes all possible integer values, and that all these values, except $H=1$ and $H=2$, may be attained in a nontrivial manner, that is such that $a \neq b$.

Corollary 5. The integers $a$ and $b$ making the harmonic mean, arithmetic mean and the geometric mean integer valued are precisely those of the form

$$
a=2 t \alpha^{2}\left(\alpha^{2}+\beta^{2}\right), \quad b=2 t \beta^{2}\left(\alpha^{2}+\beta^{2}\right),
$$

and the form

$$
\begin{aligned}
& a=t(2 \alpha+1)^{2}\left(2 \alpha+2 \alpha^{2}+2 \beta+2 \beta^{2}+1\right), \\
& b=t(2 \beta+1)^{2}\left(2 \alpha+2 \alpha^{2}+2 \beta+2 \beta^{2}+1\right),
\end{aligned}
$$

where $\alpha, \beta$ and t are positive integers.

Proof. By Theorem 3, (a,b) must belong to the class (2) or (3). By inspection, the latter is directly seen to generate integer values of $A$. But (2) gives integer values of $A$ only if both $p$ and $q$ are odd (for which (2) may be written in the form (3)) or both even, or $t$ is even. In any case, if both $H$ and $A$ are integers, we end up with the form

$$
a=2 t p(p+q), \quad b=2 t q(p+q),
$$

and the form

$$
a=t(2 p+1)(p+q+1), \quad b=t(2 q+1)(p+q+1) .
$$

Using that the product $G^{2}=a b$ is a perfect square if and only if the integers $a$ and $b$ are of the form $a=\tau r_{1}^{2}, b=\tau r_{2}^{2}$, we finally end up with the above representation. This completes the proof. 
Concerning the remaining two power means for which $k$ is an integer we have the following result:

Theorem 6. Let $t \geq 0$ be integer valued and let $x_{1}$ and $y_{1}$ denote integers of the form

$$
x_{1}=\left|p^{2}-2 p q-q^{2}\right|, \quad y_{1}=\left|p^{2}+2 p q-q^{2}\right|,
$$

for some integers $p$ and $q$. The integers $a$ and $b$ making $P_{2}$ integer valued are precisely those of the form

$$
a=t x_{1}, \quad b=t y_{1}
$$

(in this case $P_{2}=t\left(p^{2}+q^{2}\right)$ ). The integers $a$ and $b$ making $P_{-2}$ integer valued are precisely those of the form

$$
a=t\left(p^{2}+q^{2}\right) x_{1}, \quad b=t\left(p^{2}+q^{2}\right) y_{1}
$$

(in this case $\left.P_{-2}=t x_{1} y_{1}\right)$. Moreover, the integers $a$ and $b$ making all the power means $P_{-2}, H, A$ and $P_{2}$ integer valued are precisely those of the form

$$
\begin{aligned}
a & =t\left(p^{2}+q^{2}\right) x_{1} \frac{x_{1}+y_{1}}{2}, \\
b & =t\left(p^{2}+q^{2}\right) y_{1} \frac{x_{1}+y_{1}}{2} .
\end{aligned}
$$

In the representations above it is enough to use numbers $p, q$ which are coprime and have opposite parity (one odd, the other even).

Proof. If $y^{2}, z^{2}, x^{2}$ is a primitive 3 -element arithmetic progression of squares, that is $\operatorname{gcd}\left(y^{2}, z^{2}, x^{2}\right)=1$ and $z^{2}-y^{2}=x^{2}-z^{2}$, i.e.

$$
x^{2}+y^{2}=2 z^{2}
$$

then it is always possible to find two numbers $p, q$ of opposite parity (one odd, the other even) and coprime such that

$$
x^{2}=\left(p^{2}-2 p q-q^{2}\right)^{2}, \quad y^{2}=\left(p^{2}+2 p q-q^{2}\right)^{2}, \quad z^{2}=\left(p^{2}+q^{2}\right)^{2},
$$

(see e.g. [5, pp. 437-438]). Conversely, by inspection we see that $x^{2}+y^{2}=2 z^{2}$ for all squares of the form (14) and for any integers $p, q$. Thus, (9) follows by noting that $a^{2}+b^{2}=2 P_{2}^{2}$.

In the rest of the proof we assume that $P_{-2}$ is an integer. Since

$$
a^{2}+b^{2}=2\left(\frac{a b}{P_{-2}}\right)^{2},
$$

we see that $a b / P_{-2}$ is an integer. Hence, $x=a, y=b$ and $z=a b / P_{-2}$ satisfy (13). Putting $x=d x_{1}, y=d y_{1}$ and $z=d z_{1}$, where $x_{1}, y_{1}, z_{1}$ are positive integers and $d=$ $\operatorname{gcd}(x, y, z)$ (note that by (13) we also have that $d=\operatorname{gcd}(x, z)=\operatorname{gcd}(y, z))$, we obtain that 
$d x_{1} y_{1}=P_{-2} z_{1}$, and since $\operatorname{gcd}\left(x_{1} y_{1}, z_{1}\right)=1$, this shows that $d=t z_{1}$ for some integer $t$. Using that $x_{1}, y_{1}, z_{1}$ can be parametrized as in (14), i.e.

$$
x_{1}=\left|p^{2}-2 p q-q^{2}\right|, \quad y_{1}=\left|p^{2}+2 p q-q^{2}\right|, \quad z_{1}=p^{2}+q^{2},
$$

we finally obtain the representation (10) (by inspection we also see that (10) makes $P_{-2}$ integer valued for any integers $p, q)$. Since

$$
(a, b)=d\left(x_{1}, y_{1}\right)=t z_{1}\left(x_{1}, y_{1}\right)
$$

also satisfies (9), we know that $P_{2}$ also is an integer. It is easy to see that $x_{1}, y_{1}$ and $z_{1}$ are odd numbers. Hence, $A=d\left(x_{1}+y_{1}\right) / 2$ is also an integer. If, in addition, $H$ is an integer, then

$$
a=T(2 P+1)(P+Q+1), \quad b=T(2 Q+1)(P+Q+1),
$$

for some integers $T, P$ and $Q$ by (3). Moreover, according to the proof of Theorem 3 we can combine these integers in such a way that $2 P+1$ and $2 Q+1$ become relatively prime, i.e. such that

$$
x_{1}=\left|p^{2}-2 p q-q^{2}\right|=2 P+1, \quad y_{1}=\left|p^{2}+2 p q-q^{2}\right|=2 Q+1
$$

(note that (2) is of the same form as (17) due to the fact that both $x_{1}$ and $y_{1}$ are odd numbers). Thus,

$$
P+Q+1=\frac{x_{1}+y_{1}}{2} \text {. }
$$

Hence, by (17) we obtain that

$$
a=T x_{1} \frac{x_{1}+y_{1}}{2}, \quad b=T y_{1} \frac{x_{1}+y_{1}}{2},
$$

so according to (16),

$$
T \frac{x_{1}+y_{1}}{2}=t\left(p^{2}+q^{2}\right) .
$$

Since $p$ and $q$ are coprime, so are $u=\left(x_{1}+y_{1}\right) / 2$ and $v=p^{2}+q^{2}$. To see this, first observe that according to (18) $u$ takes either the forms $u= \pm\left(p^{2}-q^{2}\right)$ or $u=2 p q$. By adding and subtracting $u= \pm\left(p^{2}-q^{2}\right)=k r$ and $v=k s$, we obtain that $2 p^{2}=k(s \pm r)$, $2 q^{2}=k(s \mp r)$, which shows that $k=1$ or 2 , since $p^{2}$ and $q^{2}$ are coprime. But the latter possibility is excluded since $z_{1}=v=k s$ is odd.

$$
\begin{aligned}
& k s+k r=p^{2}+q^{2} \pm 2 p q=(p \pm q)^{2}, \\
& k s-k r=p^{2}+q^{2} \mp 2 p q=(p \mp q)^{2} .
\end{aligned}
$$

Similarly, by adding and subtracting $u=2 p q=k r$ and $v=k s$, we obtain that $k(s+r)=$ $(p+q)^{2}$ and $k(s-r)=(p-q)^{2}$, which give the solutions $2 p=\sqrt{k}(\sqrt{s+r} \pm \sqrt{s-r})$, $2 q=\sqrt{k}(\sqrt{s+r} \mp \sqrt{s-r})$, i.e. $4 p^{2}=k(\sqrt{s+r} \pm \sqrt{s-r})^{2}, 4 q^{2}=k(\sqrt{s+r} \mp$ $\sqrt{s-r})^{2}$, and, as above, we conclude that $k=1$. Since $\left(x_{1}+y_{1}\right) / 2$ and $p^{2}+q^{2}$ are coprime, (12) gives that $T=\tau\left(p^{2}+q^{2}\right)$ for some integer $\tau$, and (11) and (12) follows. Moreover, by inspection we also see that this representation yields integer values of these four means for any integers $p, q$. This completes the proof. 
Remark 7. By Corollary 5 and Theorem 6 we have a representation of all pairs $(a, b)$ making $H, G, A$ and $P_{-2}, H, A$ and $P_{2}$ integer valued, respectively. It is therefore natural to ask the question: What integers $a \neq b$ make all of the power means $P_{-2}, H, G, A$ and $P_{2}$ integer valued? The answer is: None! In fact, there are no integers $a \neq b$ making both $G$ and $P_{2}$ (or $P_{-2}$ ) integer valued. Indeed, if $a \neq b$ is of the form (9) then $a^{2}+b^{2}=2 w^{2}$, where $w=P_{2}$. Without loss of generality we may assume that $\operatorname{gcd}(a, b)=1$. If $G$ also is an integer, then $a$ and $b$ have to be perfect squares $a=u^{2}$ and $b=v^{2}$. Hence,

$$
u^{4}+v^{4}=2 w^{2} .
$$

But this equation has no nontrivial integer solution.

Remark 8. The fact that (20) has no nontrivial integer solution has been known for centuries (see [5] and [9]). Concerning the solvability of the more general equation $u^{p}+v^{p}=2 w^{2}$ it was recently proved in [8] that there are no nontrivial integer solution such that $\operatorname{gcd}(u, v, w)=1$ if $p \geq 7$ is a prime number. In the cases $p=2$ and $p=3$ there are infinitely many solutions. For both cases we are in the so called "spherical case" of the generalized Fermat equation $A x^{p}+B y^{q}=C z^{r}$, i.e. when $1 / p+1 / q+1 / r>1$ (see [1]). The case $p=5$ was treated in [2] where it was shown that $(3,-1,11),(-1,3,11)$ are the only nontrivial solutions such that $\operatorname{gcd}(u, v, w)=1$.

Remark 9. In this paper we are mainly interested in cases when the order $k$ of the power mean $P_{k}$ itself is an integer. However, an interesting problem is to find all real values of $k$ making $P_{k}=P_{k}(a, b)$ integer valued for some integers $a \neq b$. Even though we do not have the solution to this problem, it is easy to see that the number of such values is infinite, in contrast to the case when $k$ is integer valued. Indeed, let $m$ be a positive integer. Then, for any odd numbers $a$ and $b$ we have that

$$
\left(a^{m}\right)^{1 / m}+\left(b^{m}\right)^{1 / m}=2\left(c^{m}\right)^{1 / m},
$$

where $2 c=a+b$. Hence, $P_{1 / m}\left(a^{m}, b^{m}\right)=c^{m}$. Similarly, by putting $a=p(p+q)$, $b=q(p+q)$, where $p$ and $q$ are any positive integers, we obtain that

$$
\left(a^{m}\right)^{1 / m}+\left(b^{m}\right)^{1 / m}=2\left(\frac{a^{m} b^{m}}{r^{m}}\right)^{1 / m},
$$

where $r=2 p q$. Accordingly, by (1), we obtain that $P_{-1 / m}\left(a^{m}, b^{m}\right)=r^{m}$.

Acknowledgements. We thank Professor Kenneth A. Ribet at University of California, Berkeley, for some interesting discussions and for showing us a simple proof that the equation $u^{4}+v^{4}=2 w^{2}$ is unsolvable [11]; a fact which led to the interesting conclusion given in Remark 7. In addition we want to thank Professor Alain Kraus, Professor Luïc Merel and Dr. Wilfrid Ivorra at Université Pierre et Marie Curie for valuable information concerning the equation $u^{p}+v^{p}=2 w^{2}$. Finally, we want to thank an anonymous referee for suggestions which have improved the presentation of the paper. 


\section{References}

[1] Beukers, F.: The Diophantine equation $A x^{p}+B y^{q}=C z^{r}$. Duke Math. J. 91 (1998)1, 61-88.

[2] Bruin, N.: Chabauty methods and covering techniques applied to generalized Fermat equations. PhDthesis, University of Leiden, 1999.

[3] Bullen, P.S.; Mitrinovic, D.S.; Vasic, P.M.: Means and their inequalities. D. Reidel Publishing Company Dordrecht, 1988.

[4] Darmon, H.; Merel, L.: Winding quotients and some variants of Fermat's Last Theorem. J. Reine Angew. Math. 490 (1997), 81-100.

[5] Dickson, L.E.: History of the Theory of Numbers, Vol. 2: Diophantine Analysis. New York, Dover 2005.

[6] Denés, P.: Über die Diophantische Gleichung $x^{l}+y^{l}=c z^{l}$. Acta Math. 88 (1952), 241-251.

[7] Hardy, G.H.; Littlewood, J.E.; Pólya, G.: Inequalities. Cambridge University Press, 1934.

[8] Ivorra, W.: Sur les équations $x^{p}+2^{\beta} y^{p}=z^{2}$ et $x^{p}+2^{\beta} y^{p}=2 z^{2}$. Acta Arith. 108 (2003), 327-338.

[9] Ribenboim, P.: Catalan's conjecture. Are 8 and 9 the only consecutive powers? Academic Press Inc., Boston MA 1994.

[10] Ribet, K.: On the equation $a^{p}+2^{\alpha} b^{p}+c^{p}=0$. Acta Arith. 79 (1997)1, 7-16.

[11] Ribet, K.: Private communication, 2007.

\section{Ralph Høibakk}

Narvik University College

N-8505 Narvik, Norway

Dag Lukkassen

Narvik University College

P.O. Box 385

N-8505 Narvik, Norway

and

Norut Narvik

P.O. Box 250

N-8504 Narvik, Norway

e-mail: Dag.Lukassen@hin.no 